\title{
Test-retest reliability of static and dynamic motor fatigability protocols using grip and pinch strength in typically developing children
}

Citation for published version (APA):

Brauers, L., Smeets, R., Feys, P., Bastiaenen, C., Klingels, K., \& Rameckers, E. (2021). Test-retest reliability of static and dynamic motor fatigability protocols using grip and pinch strength in typically developing children. European Journal of Pediatrics, $180(8), 2505-2512$. https://doi.org/10.1007/s00431021-04033-y

Document status and date:

Published: 01/08/2021

DOI:

10.1007/s00431-021-04033-y

Document Version:

Publisher's PDF, also known as Version of record

Document license:

Taverne

Please check the document version of this publication:

- A submitted manuscript is the version of the article upon submission and before peer-review. There can be important differences between the submitted version and the official published version of record.

People interested in the research are advised to contact the author for the final version of the publication, or visit the DOI to the publisher's website.

- The final author version and the galley proof are versions of the publication after peer review.

- The final published version features the final layout of the paper including the volume, issue and page numbers.

Link to publication

\footnotetext{
General rights rights.

- You may freely distribute the URL identifying the publication in the public portal. please follow below link for the End User Agreement:

www.umlib.nl/taverne-license

Take down policy

If you believe that this document breaches copyright please contact us at:

repository@maastrichtuniversity.nl

providing details and we will investigate your claim.
}

Copyright and moral rights for the publications made accessible in the public portal are retained by the authors and/or other copyright owners and it is a condition of accessing publications that users recognise and abide by the legal requirements associated with these

- Users may download and print one copy of any publication from the public portal for the purpose of private study or research.

- You may not further distribute the material or use it for any profit-making activity or commercial gain

If the publication is distributed under the terms of Article $25 \mathrm{fa}$ of the Dutch Copyright Act, indicated by the "Taverne" license above, 


\title{
Test-retest reliability of static and dynamic motor fatigability protocols using grip and pinch strength in typically developing children
}

\author{
Lieke Brauers $^{1,2}$ (D) Rob Smeets $^{2} \cdot$ Peter Feys $^{1} \cdot$ Caroline Bastiaenen $^{4} \cdot$ Katrijn Klingels $^{1} \cdot$ Eugene Rameckers $^{1,2,3,5}$
}

Received: 30 July 2020 / Revised: 10 March 2021 / Accepted: 12 March 2021 / Published online: 19 April 2021

(C) The Author(s), under exclusive licence to Springer-Verlag GmbH Germany, part of Springer Nature 2021

\begin{abstract}
Upper limb motor fatigability is an important debilitating factor for activities of daily living in clinical pediatric populations. However, the reliability of fatigability protocols in these populations is currently unknown. Therefore, the current study investigates test-retest reliability of a static and dynamic motor fatigability protocol for grip and pinch strength in typically developing children (TDC). Eighty-nine TDC (35 boys, 54 girls; mean age 10 years 11 months) used a grip and pinch dynamometer for static (sustained) and dynamic (repeated) contractions during 30s. For static motor fatigability (SFI), mean (Fmean) and SD (Fvar) of force were calculated, and for dynamic motor fatigability, F mean and number of peaks (Npeaks) were calculated. Intraclass correlation coefficients (ICC) were calculated. ICCs of F mean in static and dynamic motor fatigability were high (ICC: 0.940.96 and $0.91-0.98$ ). ICCs were moderate to high for F var (ICC: 0.67-0.85). The SFI showed moderate ICCs (ICC: 0.69-0.77). ICCs were moderate to high for N peaks (ICC: 0.78-0.91).

Conclusion: The results suggest that static and dynamic motor fatigability in for grip and pinch can be used reliably in TD children aged 6-18 years.
\end{abstract}

\section{What is Known:}

- Psychometric properties of motor fatigability protocols using grip and pinch in children are lacking.

-Motor fatigability in grip and pinch is an important debilitating symptom in multiple neurologic populations.

What is New:

- Static fatigability can be investigated using a 30-s maximum sustained grip strength protocol in children.

-Dynamic fatigability can be investigated using a 30-s maximum repeated grip strength protocol in children.

Katrijn Klingels and Eugene Rameckers contributed equally to this work.

Communicated by Gregorio Paolo Milani

Lieke Brauers

lieke.brauers@uhasselt.be

Rob Smeets

r.smeets@maastrichtuniversity.nl

Peter Feys

peter.feys@uhasselt.be

Caroline Bastiaenen

chg.bastiaenen@maastrichtuniversity.nl

Katrijn Klingels

katrijn.klingels@uhasselt.be

Eugene Rameckers

eugene.rameckers@uhasselt.be
1 REVAL - Rehabilitation Research Center, Faculty of Rehabilitation Sciences, Hasselt University, Martelarenlaan 42, 3500 Hasselt, Belgium

2 Department of Rehabilitation Medicine, Research School CAPHRI, Maastricht University, Maastricht, Netherlands

3 Master of Specialized Physical Therapy, AVANSplus, Breda, Netherlands

4 Department of Epidemiology, Research Line: Function, Participation \& Rehabilitation, CAPHRI, Maastricht University, Maastricht, Netherlands

5 Center of Expertise, Adelante Rehabilitation Center, Valkenburg, Netherlands 
Keywords Upper limbs · Pediatric rehabilitation $\cdot$ Motor fatigability

$\begin{array}{ll}\text { Abbreviations } \\ \text { AUC } & \begin{array}{l}\text { Area under the curve } \\ \text { CI }\end{array} \\ \text { Confidence Interval } \\ \text { CPSIN } & \begin{array}{l}\text { Consensus-based standards for the selection of } \\ \text { health measurement instrument }\end{array} \\ \text { CP } & \text { Cerebral palsy } \\ \text { DFI } & \text { Dynamic fatigue index } \\ \text { Fmean } & \text { Mean force } \\ \text { Fvar } & \text { Standard deviation of force } \\ \text { HAUC } & \text { Hypothetical area under the curve } \\ \text { ICC } & \text { Intraclass correlation coefficient } \\ \text { Kg } & \text { Kilograms } \\ \text { LOA } & \text { Limits of agreement } \\ \text { MS } & \text { Multiple sclerosis } \\ \text { MVC } & \text { Maximum voluntary contraction } \\ \text { rMVC } & \text { Repeated maximum voluntary contraction } \\ \text { SD } & \text { Standard deviation } \\ \text { SDD } & \text { Smallest detectable difference } \\ \text { SEM } & \text { Standard error of measurement } \\ \text { SFI } & \text { Static fatigue index } \\ \text { SMVC } & \text { Sustained maximum voluntary contraction } \\ \text { TD } & \text { Typically developing }\end{array}$

\section{Introduction}

Fatigue may have a detrimental impact on everyday functioning in patients with a neurological disorder [1-4]. In young adults with cerebral palsy (CP), general fatigue (76\%), pain (88\%), and joint deformities (86\%) have been reported as major health problems [5]. In children with unilateral CP, in whom one side of the body is affected, perceived fatigue is associated with decreased motor performance and strength [4]. Also, in other pediatric populations, such as pediatric stroke, juvenile idiopathic arthritis, and Duchenne muscular dystrophy, the presence of fatigue in general has been reported and is known to affect activities of daily living and quality of life $[4,6,7]$.

In this study, fatigue is defined according to the taxonomy of Kluger et al. (2013) [8, 9]. Fatigue is divided into "trait fatigue," constant and independent of time or daily activities, and "state fatigue" (fatigability), dependent on daily activities and therefore fluctuating in time. Here, "state fatigue" or fatigability is defined as "a decline in one or more aspects of performance during continuous execution of a prolonged task or comparing performance on a probe task before and immediately after prolonged execution of a separate fatigueinducing task" $[8,10]$. Fatigability can be further divided into two domains, cognitive (using cognitive tasks) and motor (using motor tasks). In this study, we focus on the motor domain, defined as "the magnitude or rate of change of motor performance on an objectively measured reference criterion after any type of voluntary activity or exercise" $[8,10]$. Furthermore, static motor fatigability and dynamic motor fatigability can be distinguished, depending on the type of task performed [11]. For static motor fatigability, persons perform a sustained maximal voluntary contraction (sMVC) over a given period of time, whereas in dynamic motor fatigability, persons perform repeated maximal voluntary contractions (rMVC) over a given period of time [11].

In pediatric populations, motor fatigability has been investigated in children with $\mathrm{CP}$ using several measurement protocols with different outcome measures such as the static fatigue index (SFI); EMG measures; and muscle endurance for the hands, elbows, and shoulders [12-17]. These studies report conflicting results which might be influenced by the lack of research on clinimetric properties of these protocols in pediatric populations [12-17]. Further research in this area is warranted.

In adults with multiple sclerosis (MS), static motor fatigability is measured using a 30-s sustained strength task and dynamic motor fatigability using a 30 -s repeated strength task $[11,18]$. For static motor fatigability, the SFI is calculated by comparing the actual area under the force-time curve (AUC) to a hypothetical area under the curve (HAUC), if there was no fatigability. A higher SFI represents a higher static motor fatigability $[11,18]$. As well as SFI, other parameters may be relevant in quantifying fatigability in children such as the mean force and the standard deviation of force. For static motor fatigability, mean force and standard deviation (SD) of force can be calculated in the first and third parts of the 30 -s force-time curve. Static motor fatigability could then present as a decrease in mean force and/or an increase in SD of force with time.

Previous research in MS patients has quantified that dynamic motor fatigability was measured using the dynamic fatigue index (DFI) [13]. The DFI is calculated as the ratio between the first three and last three peak forces in the 30-s repeated strength task. However, for DFI, mean force and the number of peaks may also be relevant. Here, the $30 \mathrm{~s}$ period can again be divided into three parts, with the mean force and the number of peaks in each part being calculated. A decrease in force and/or a decrease in number of peaks between the first and third parts indicates a higher motor fatigability.

Despite that several protocols are proposed for measuring static and dynamic motor fatigability in pediatric populations, data on reliability is lacking [12-17]. A clinimetrically sound protocol in typically developing (TD) children is warranted to develop norm-values for motor fatigability in children. This in turn could support the understanding and interpretation of data of pediatric populations such as cerebral palsy, juvenile idiopathic arthritis, Duchenne muscular dystrophy, and spinal muscular 
atrophy regarding the presence and severity of motor fatigability. The static and dynamic motor fatigability protocols that will be investigated in the current study are mainly based on protocols found to be reliable in MS patients $[2,11,18-20]$. The aim of this paper is to investigate the test-retest reliability of two new motor fatigability protocols in TD children.

\section{Methods}

\section{Participants}

Typically developing children from different primary schools, high schools, youth movements, and friends and family of the researchers, in Belgium were invited to participate between December 2018 and December 2019. Numbers were based on the consensus-based standards for the selection of health measurement instruments (COSMIN) guidelines which state that at least 100 children need to be included for a good study design [21]. Children were included if they were (1) aged between 6 and 18 years, (2) cognitively capable of understanding the instructions, (3) Dutch speaking, and (4) willing to participate which was assessed using dialog with the child prior to testing. Parents were asked to fill in a questionnaire to identify health-related problems, such as neurological, orthopedic, or cognitive disorders. Children were excluded if they suffered any type of upper limb motor disorder (neurological and/or orthopedic) or a cognitive disorder. Children were excluded if they suffered any type of upper limb motor disorder (neurological and/or orthopedic) or a cognitive disorder.

Information on age, gender, and self-reported preferred hand was gathered. The dominant hand was defined as the hand with which children wrote or colored. Children were asked not to participate in intensive fatigable exercises of the upper limb in the day before and on the day of testing. Children and/or parents gave written informed consent prior to the measurements. This research was approved by the Medical Ethical Committee of Hasselt University (CME2018/069).

\section{Measurements}

Each child was measured twice by the same tester, with a minimum of $24 \mathrm{~h}$ and a maximum of 1 week between measurements. Children were seated in a chair with their feet flat on the floor, back against the backrest, shoulders in neutral position, and elbows in 90 degrees. There was no support of the shoulder. The forearm and elbow were leaning on an armrest, and wrist in neutral position [12]. The grip meter and pinch meter were not additionally supported by the tester. Positioning of the children was always standardized across tests. Five testers (physiotherapists) performed the measurements. All were trained by the lead investigator (LB) who provided a detailed, standardized protocol. Furthermore, a detailed log was written after the testing of each child, noting any uncertainties during measurements. All grip and pinch strength and fatigability measurements were performed with the Biometrics E-LINK H500 Hand Kit (Biometrics Ltd., UK). Forces are given in kilograms $(\mathrm{kg})$. The handle position of the E-LINK H500 Hand Kit can be adjusted to the size of the hand. There are three different positions, and the correct position was determined so that the end of the handlebar approximates the distal end of the metacarpal. The grip meter and pinch meter were not additionally supported by the tester.

\section{Measurement procedures}

First, children performed three maximum voluntary contractions (MVC) with the grip and pinch dynamometer with each hand. After each MVC, a 30-s rest was provided. The mean of the three trials was used for further analyses.

Second, static motor fatigability was measured during a 30s sustained maximal contraction with the grip and pinch dynamometer. Both hands were tested. Instructions were standardized across testers, and children were asked to squeeze as hard as possible for $30 \mathrm{~s}$, while visual feedback on the remaining time was provided. The execution was defined as successful if the children reached the peak force within the first $10 \mathrm{~s}$ of the measurement. This ensured the ability to correctly calculate the outcome measurements.

Third, dynamic motor fatigability was assessed also over a 30-s time period. Children squeezed repeatedly as hard and fast as possible. Again, verbal instructions and encouragement were standardized across testers and every time children were shown visual feedback on the remaining time. The measurement was defined as successful if the children squeezed repeatedly at any pace over the entire $30 \mathrm{~s}$.

For both static and dynamic motor fatigability, no test session was scheduled because in that way, motor fatigability could be present more than was actually the case. Also, because of the easy nature of both tasks, a test session would not be necessary.

\section{Outcome measures}

\section{Fatigability parameters}

Static motor fatigability was quantified by calculating three outcome measures: mean force $\left(\mathrm{F}_{\text {mean }}\right)$, SD of force $\left(\mathrm{F}_{\mathrm{var}}\right)$, and the static fatigue index (SFI) [11]. To calculate these, peak force within the first $10 \mathrm{~s}$ was identified, and the curve prior to peak force was excluded from calculation. For calculating the outcome measures, $F_{\text {mean }}$ and $F_{\text {var }}$, the remaining time was divided into three equal parts. Within the first and the third parts, mean force was calculated $\left(\mathrm{F}_{\text {mean } 1}, \mathrm{~F}_{\text {mean } 3}\right)$, as well as the SD of force $\left(\mathrm{F}_{\mathrm{var}}, \mathrm{F}_{\mathrm{var} 3}\right)$ (Fig. 3a). Test-retest reliability 
Fig. 1 a Calculation of $\mathrm{F}_{\text {mean }}$ and $\mathrm{F}_{\mathrm{var}}$ within three time slots. Continuous horizontal line, $\mathrm{F}_{\text {mean }}$; dashed horizontal line, $\mathrm{F}_{\mathrm{var}} \cdot \mathbf{b}$ Schematic overview of the areas used to calculate the static fatigue index. AUC, area under the curve; HAUC, hypothetical area under the curve

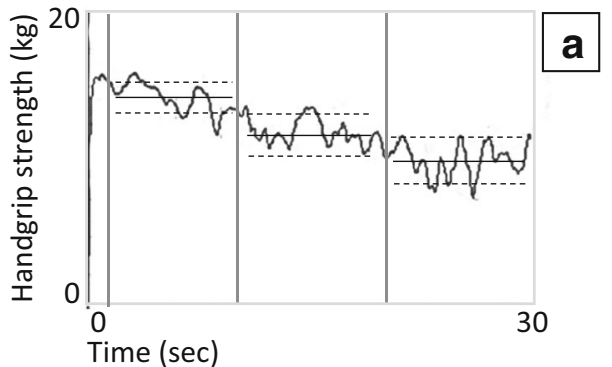

was calculated for $F_{\text {mean }}$ and $F_{\text {var }}$ for the first and third parts of the force-time curve.

To calculate SFI, as in the study of Surakka et al. (2004), two areas were identified: first, an area under the force-time curve (AUC) and, second, a hypothetical area under the curve (HAUC) mimicking a situation where strength would have been sustained at maximum level during the trial [18]. The SFI was then calculated by using the following equation: $\mathrm{SFI}=100 \% \times 1-($ AUC/HAUC) $[11]$ (Fig. 1b). Following this equation, a higher SFI represents more fatigability in grip and pinch.

To calculate dynamic fatigability, two outcome measures were used, $\mathrm{F}_{\text {mean }}$ and $\mathrm{N}_{\text {peaks. }}$. First, the 30 -s force-time curve was divided into three equal parts. Within the first and third parts, the mean forces were calculated $\left(\mathrm{F}_{\text {mean } 1}, \mathrm{~F}_{\text {mean3 } 3}\right)$ and the number of peaks counted ( $\mathrm{N}_{\text {peaks } 1}, \mathrm{~N}_{\text {peaks } 3}$ ) (Fig. 2). Test-retest reliability of dynamic fatigability was calculated for $\mathrm{F}_{\text {mean }}$ and $\mathrm{N}_{\text {peaks }}$ in the first and third parts of the force-time curve.

\section{Statistical analysis}

Descriptive statistics are presented for the participants (age, gender, handedness) and are presented with a mean (SD)/

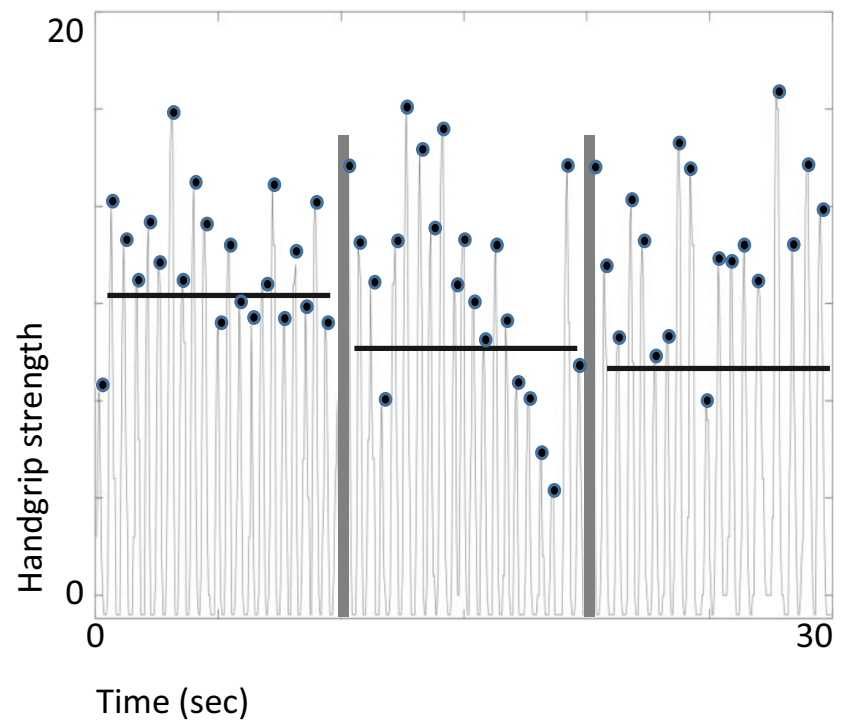

Fig. 2 Schematic representations of outcome measures for dynamic fatigability. Bullets, peaks; horizontal line, $\mathrm{F}_{\text {mean }}$ median (IQR), whichever appropriate, or number of participants with that characteristic (gender, handedness).

To investigate reliability and agreement, three steps in the analyses were used:

\section{Reliability}

First, Bland-Altman plots with limits of agreement (LOA) were used to investigate absolute agreement between the two measurements $[22,23]$. The LOA were established by the following: mean difference $\pm 1.96 * \mathrm{SD}$ of the difference between the two test measurements.

Second, Bland-Altman plots were visually checked for heteroscedasticity. To confirm or reject this, Kendall's tau $(\tau)$ was used to indicate the correlation between the absolute difference and the corresponding means. Kendall's $\tau \leq 0.1$ indicates that the use of absolute analysis such as ICCs is appropriate [22, 23].

The last step in investigating how well patients can be distinguished from each other was calculated using ICCs and $95 \%$ confidence intervals (CI) with a two-way random model [21]. ICCs were calculated in both the entire group of children, aged 6-18 years, as well as two subgroups: 611 years $(n=35)$ and $12-18$ years $(n=56)$. ICCs were interpreted as follows: $<0.40$, low reliability; $0.40-0.79$, moderate reliability; and $0.80-1.00$, good reliability [24]. Given the explorative character of the study, no hypotheses were formed regarding the expected magnitude of the ICC values.

Statistical analysis was performed using SPSS Statistics 25 (IBM SPSS Statistics 25, OIBM Armonk, NY, US).

\section{Results \\ Participants}

A total of 108 children responded to the invitation and were included in to the study. For static motor fatigability and dynamic motor fatigability, two children did not have successful measurements due to technical issues, and 17 did not attend the second testing. A total of 89 children with complete datasets were included in the analysis (median age, 11 years 


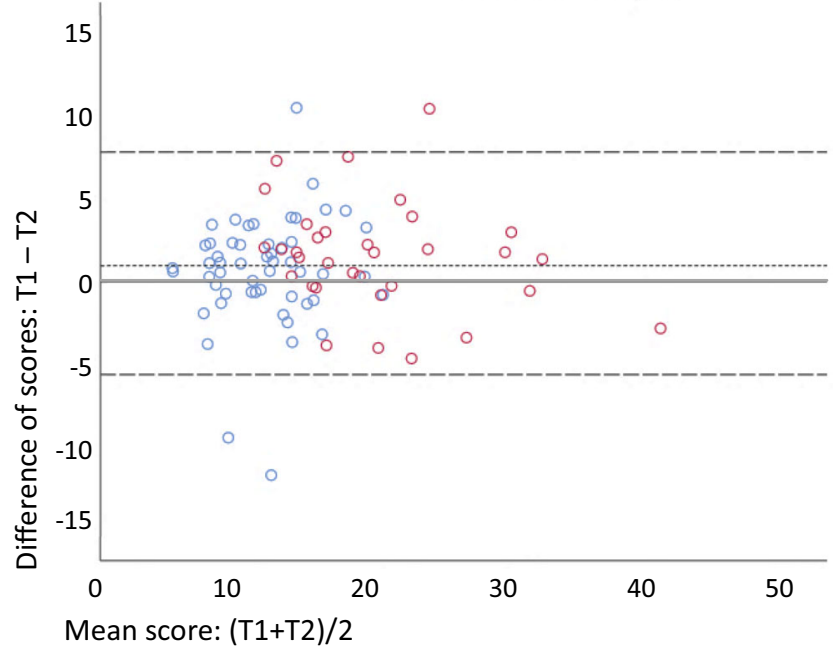

Fig. 3 Bland-Altman plot of measurements 1 and 2 of the handgrip task for the dominant hand. The middle lines show the mean difference between the two measures (smaller dash) and zero (solid line), and the upper and lower dashed lines show the limits of agreement. The $x$-axis shows the mean of both measures, and the $y$-axis shows the difference between both measurements. The blue dots show the younger children (6-11 years), and the red dots show the older children (12-18 year)

(IQR: 6); mean age, 10.90 (SD) 3,62); range, 6-18 years) of which 35 were boys and 54 girls. Fifty-six children were aged between 6 and 11 years and 35 between 12 and 18 years; 79 children were right-handed.

\section{Bland-Altman plots}

For all outcome measures, Bland-Altman plots were performed and LOAs calculated. An example of the BlandAltman plot for $\mathrm{F}_{\text {mean } 1}$ of static motor fatigability in the dominant hand is shown in Figure $3[22,23]$. The smaller dashed line shows the mean systematic difference between T1 and T2 measurements which is close to 0 indicating a small systematic error [21]. The upper and lower dashed lines show the random error. In this case, more than $95 \%$ of cases fall between the dashed lines indicating a normal distribution. This figure shows that the variability in the difference between T1 and $\mathrm{T} 2$ is similar across the entire range of means between $\mathrm{T} 1$ and T2 indicating homoscedasticity. Also, the number of extreme values in the total group was small (4 out of 89 children). All Kendall's $\tau$ values were $\leq 0.1$, indicating that no cases of heteroscedasticity were identified and that no $\log$ transformations were needed. The remaining plots of all outcome measures are included in the supplementary material.

\section{Test-retest reliability in static motor fatigability}

All results regarding the reliability analyses are shown in Table 1. Details on ICC values in 6-11-year-old children and 12-18-year-old children are presented in Supplementary Tables 2 and 3. For the grip meter, ICCs of the mean force ( $\mathrm{F}_{\text {mean } 1}$ and $\mathrm{F}_{\text {mean } 3}$ ) were 0.95 and 0.96 in the dominant and 0.95 and 0.94 in the non-dominant hand respectively, indicating good reliability. For the variability (SD: $\mathrm{F}_{\mathrm{Var} 1}$ and $\mathrm{F}_{\mathrm{Var} 3}$ ), ICCs were 0.74 and 0.81 in the dominant and 0.82 and 0.67 in the non-dominant hand, respectively, indicating moderate to good reliability. ICC ranges were similar for both 6-11 year old children and 12-18 year old children.

For the pinch meter in the dominant hand, ICCs of the mean force $\left(\mathrm{F}_{\text {mean } 1}\right.$ and $\left.\mathrm{F}_{\text {mean3 } 3}\right)$ were 0.95 and 0.94 ; they were the same in the non-dominant hand indicating good reliability. For the variability (SD: $\mathrm{F}_{\text {var1 }}$ and $\mathrm{F}_{\mathrm{var} 3}$ ), the ICCs were 0.71 and 0.69 in the dominant and 0.85 and 0.71 in the nondominant hand, respectively, which again indicates moderate

Table 1 Results of static and dynamic motor fatigability protocols

\begin{tabular}{|c|c|c|c|c|c|c|c|c|c|}
\hline \multirow[b]{3}{*}{ Dom hand } & \multicolumn{4}{|c|}{ Static motor fatigability } & & \multicolumn{4}{|c|}{ Dynamic motor fatigability } \\
\hline & \multicolumn{2}{|c|}{ Hand grip } & \multicolumn{2}{|c|}{ Pinch grip } & & \multicolumn{2}{|c|}{ Hand grip } & \multicolumn{2}{|c|}{ Pinch grip } \\
\hline & $\mathrm{ICC}$ & $\mathrm{CI}$ & ICC & $\mathrm{CI}$ & & ICC & $\mathrm{CI}$ & $\mathrm{ICC}$ & $\mathrm{CI}$ \\
\hline Fmean 1 & 0.95 & $0.923-0.970$ & 0.95 & $0.921-0.970$ & Fmean 1 & 0.98 & $0.961-0.984$ & 0.93 & $0.886-0.958$ \\
\hline Fmean 3 & 0.96 & $0.929-0.974$ & 0.94 & $0.905-0.963$ & Fmean 3 & 0.97 & $0.951-0.981$ & 0.91 & $0.864-0.943$ \\
\hline FVar1 & 0.74 & $0.599-0.835$ & 0.71 & $0.536-0.813$ & Npeaks1 & 0.78 & $0.548-0.876$ & 0.85 & $0.646-0.925$ \\
\hline FVar3 & 0.81 & $0.707-0.882$ & 0.69 & $0.502-0.804$ & Npeaks3 & 0.81 & $0.544-0.907$ & 0.91 & $0.848-0.940$ \\
\hline SFI & 0.69 & $0.535-0.799$ & 0.76 & $0.633-0.843$ & & & & & \\
\hline N-Dom hand & $\mathrm{ICC}$ & $\mathrm{CI}$ & ICC & $\mathrm{CI}$ & & ICC & $\mathrm{CI}$ & $\mathrm{ICC}$ & $\mathrm{CI}$ \\
\hline Fmean 1 & 0.95 & $0.923-0.970$ & 0.95 & $0.909-0.967$ & Fmean 1 & 0.97 & $0.948-0.983$ & 0.93 & $0.882-0.952$ \\
\hline Fmean 3 & 0.94 & $0.903-0.959$ & 0.94 & $0.902-0.961$ & Fmean 3 & 0.97 & $0.952-0.980$ & 0.92 & $0.876-0.947$ \\
\hline FVar1 & 0.82 & $0.722-0.883$ & 0.85 & $0.767-0.909$ & Npeaks1 & 0.89 & $0.618-0.951$ & 0.91 & $0.848-0.945$ \\
\hline FVar3 & 0.67 & $0.480-0.785$ & 0.71 & $0.534-0.815$ & Npeaks3 & 0.84 & $0.587-0.919$ & 0.90 & $0.734-0.948$ \\
\hline SFI & 0.77 & $0.648-0.848$ & 0.76 & $0.641-0.845$ & & & & & \\
\hline
\end{tabular}

Abbreviations: $N$-Dominant non-dominant, ICC intraclass correlation coefficient, $C I 95 \%$ confidence interval 
to good reliability. Again, ICC ranges in reliability were similar for both age groups.

For the SFI of the grip and the pinch meter, the ICC values ranged between 0.69 and 0.77 for both hands which indicates moderate reliability. For the 6-11-year-old children, ICCs ranged from 0.58 to 0.83 and for the 12-18-year-old children from 0.48 to 0.83 , which indicates moderate to good reliability.

\section{Test-retest reliability in dynamic motor fatigability}

For the grip meter, ICCs for $\mathrm{F}_{\text {mean } 1}$ and $\mathrm{F}_{\text {mean3 }}$ were 0.98 and 0.97 in the dominant and 0.97 and 0.97 in the non-dominant hand which indicates good reliability. For the grip meter, ICCs of $\mathrm{N}_{\text {peaks } 1}$ and $\mathrm{N}_{\text {peaks3 } 3}$ were 0.78 and 0.81 in the dominant and 0.89 and 0.84 in the non-dominant hand, respectively, indicating moderate to good reliability. For both age groups, these reliability ranges were similar for $\mathrm{F}_{\text {mean } 1}$ and $\mathrm{F}_{\text {mean3 } 3}$. However, for $\mathrm{N}_{\text {peaks } 1}$ and $\mathrm{N}_{\text {peaks } 3}$, the ICC range in the 6-11-year-old children was from $0.81-0.90$ indicating good reliability.

For the pinch meter, the ICCs of $\mathrm{F}_{\text {mean } 1}$ and $\mathrm{F}_{\text {mean3 } 3}$ were 0.93 and 0.91 in the dominant and 0.93 and 0.92 in the nondominant hand, respectively. Good reliability was also found in both age groups. For the pinch meter, the ICCs of $\mathrm{N}_{\text {peaks } 1}$ and $\mathrm{N}_{\text {peaks3 } 3}$ were 0.85 and 0.91 in the dominant and 0.91 and 0.90 in the non-dominant hand, respectively. Again, good reliability was found also in both age groups.

\section{Discussion}

/ align="center"/ align="left"/cgqizThis study investigated the test-retest reliability of two static and dynamic motor fatigability protocols in TD children. Based on the ICCs, we are confident that these protocols can be used in TD children receiving reliable data. The protocols are useful to develop a reference database to investigate the presence and severity of motor fatigability using grip and pinch in pediatric populations. Static and dynamic motor fatigability were investigated in grip and pinch strength using two 30-s maximum exertion protocols. For static motor fatigability, mean $\left(\mathrm{F}_{\text {mean }}\right)$ and $\mathrm{SD}$ $\left(\mathrm{F}_{\mathrm{var}}\right)$ of forces in the first and last thirds of the trial were calculated as well as the SFI. For dynamic motor fatigability, mean force $\left(\mathrm{F}_{\text {mean }}\right)$ and number of peaks $\left(\mathrm{N}_{\text {peaks }}\right)$ in the first and last thirds of the trial were calculated. It is the first time that this calculation method regarding static and dynamic motor fatigability is introduced in children.

Mean forces $\left(\mathrm{F}_{\text {mean }}\right)$ showed good reliability and small 95\% CI for both static and dynamic motor fatigability. For static motor fatigability, this is in line with reliability studies in the hands using peak strength measures in TD children [25, 26]. In these studies, peak force was calculated as the mean over three 3-4-s maximum voluntary contractions (MVC), instead of a sustained 30-s maximum exertion protocol, as in this study $[25,26]$. This could have had an influence on the reliability results. However, although the protocols measure different aspects of force, they both seem equally reliable in TD children.

A novelty of this study was the investigation of variability $\left(\mathrm{F}_{\text {var }}\right)$ for static motor fatigability and the number of peaks $\left(\mathrm{N}_{\text {peaks }}\right)$ for dynamic motor fatigability. Both outcome measures showed moderate to good reliability based on ICCs. However, ICCs were slightly lower and 95\% CI were wider than those of $\mathrm{F}_{\text {mean }}$ in both static and dynamic motor fatigability. Also, confidence intervals in $\mathrm{F}_{\mathrm{var}}$ and $\mathrm{N}_{\text {peaks }}$ were larger than in $F_{\text {mean. }}$. For $F_{\text {var }}$ and $N_{\text {peaks }}$, no comparable studies on reliability were found, but it is possible that ICCs were influenced by the complex calculations to receive the final outcome measures. Future research in pediatric populations needs to identify which outcome measure for static and dynamic motor fatigability is preferable based on reliability, validity and feasibility.

The SFI has been used previously to investigate static fatigability in CP patients [12], without available results on reliability. In TD children, ICC values indicated moderate reliability for all outcome measures. In healthy adults, similar reliability was found, while in MS patients, higher reliability was found compared to our study in TD children [11, 18]. Between-subject variability in relation to within subject variability is important for the level of ICC as higher variability between subjects results in higher ICCs. This could possibly explain the differences in ICCs in healthy adults, MS patients, and TD children [11,27].

In addition to calculating the ICC values for the 6-18 group, also ICC values were calculated per age range, i.e., 6-11 years and 12-18 years. Although reliability in the younger children was still moderate to good, ICC values were generally lower compared to the total group as well as the older children. These lower ICCs may be partly explained by the smaller sample size $(N=35)$, which decreased between-subject variability. Nonetheless, these lower ICCs in the younger children indicate that more caution is needed when using the protocols in this age range in comparison to older children.

Based on ICCs in both the entire group as well as in the subgroups and Bland-Altman plots, sufficient reliability and agreement can be assumed for $\mathrm{F}_{\text {mean }}, \mathrm{F}_{\text {var }}$, and $\mathrm{N}_{\text {peaks }}$ in both the first and last parts of the force-time curve. However, since in this study motor fatigability has been defined as: "the magnitude or rate of change of motor performance on an objectively measured reference criterion after any type of voluntary activity or exercise," a calculation has to be performed to measure the change between the first and last parts of the force-time curve. Surakka et al. (2004) and Schwidd et al. (1999) have used the ratio between the first and last parts of the curve to describe the change in $F_{\text {mean }}[11,18]$. We propose 
to use this ratio for the calculation of motor fatigability in different patient populations in the future.

Furthermore, current data were collected within a large age range, at several times of the day, with more than one therapist, and within in a (possibly) noisy school environment making the test condition not very standardized. This may have influenced our results on reliability. However, these test conditions were close to the clinical practice which allows generalization of the results. Another aspect that may have standardized testing further would be to consider using the Edinburgh Handedness Inventory.

Last, the small number of children in this study is not sufficient yet to use as a reference database. However, because the data showed good reliability, the data can provide as an initial step in building a database as a reference to compare TD children with other pediatric populations in a cross-sectional situation.

For future research, the next step should be to assess the reliability of these protocols for motor fatigability in patient populations. Also, the standard error of measurement (SEM) and smallest detectable difference (SDD) should be investigated to ensure the usability of these outcomes to measure change over time $[28,29]$.

To summarize, we conclude that our results indicate a moderate-to-good reliability for static and dynamic motor fatigability protocols for grip and pinch strength in TD children aged 6 to 18 years in a cross-sectional design. In the future, reliability of these protocols in other pediatric populations should be investigated.

Supplementary Information The online version contains supplementary material available at https://doi.org/10.1007/s00431-021-04033-y.

Authors' contributions LB: Medical ethics approval, writing full manuscript, data collection, data analysis, interpretation of results, finalizing manuscript

RS: Writing full manuscript, interpretation of results

PF: Writing full manuscript, interpretation of results

$\mathrm{CB}$ : Writing full manuscript, data collection, data analysis, interpretation of results

KK: Medical ethics approval, writing full manuscript, data collection, data analysis, interpretation of results

ER: Medical ethics approval, writing full manuscript, data collection, data analysis, interpretation of results

Data availability N/A.

Code availability N/A.

\section{Declarations}

Ethics approval This study received ethics approval for performing tests in children at Hasselt University (CME2018/069).
Consent to participate Written informed consent was provided by participants (and parents).

Consent for publication Written informed consent was provided by participants (and parents).

Informed consent Informed consent was obtained from all individual participants included in the study.

Conflict of interest The authors declare no competing interests.

\section{References}

1. Aldughmi M, Bruce J, Siengsukon CF (2016) Relationship between fatigability and perceived fatigue measured using the neurological fatigue index in people with multiple sclerosis. Int J MS Care 19(5):232-239

2. Severijns D, Lamers I, Kerkhofs L, Feys P (2015) Hand grip fatigability in persons with multiple sclerosis according to hand dominance and disease progression. J Rehabil Med 47(2):154-160

3. Tseng BY, Kluding P (2009) The relationship between fatigue, aerobic fitness, and motor control in people with chronic stroke: a pilot study. J Geriatr Phys Ther (2001) 32(3):97-102

4. Wrightson JG, Zewdie E, Kuo HC, Millet GY, Kirton A (2019) Fatigue in children with perinatal stroke: clinical and neurophysiological associations. Dev Med Child Neurol

5. Hilberink SR, Roebroeck ME, Nieuwstraten W, Jalink L, Verheijden JM, Stam HJ (2007) Health issues in young adults with cerebral palsy: towards a life-span perspective. J Rehabil Med 39(8):605-611

6. Armbrust W, Lelieveld $\mathrm{OH}$, Tuinstra $\mathrm{J}$ et al (2016) Fatigue in patients with juvenile idiopathic arthritis: relationship to perceived health, physical health, self-efficacy, and participation. Pediatr Rheumatol Online J 14(1):65

7. El-Aloul B, Speechley KN, Wei Y, Wilk P, Campbell C (2020) Fatigue in young people with Duchenne muscular dystrophy. Dev Med Child Neurol 62(2):245-251

8. Kluger BM, Krupp LB, Enoka RM (2013) Fatigue and fatigability in neurologic illnesses Proposal for a unified taxonomy. Neurology 80:8

9. Van Geel F, Moumdjian L, Lamers I, Bielen H, Feys P (2019) Measuring walking-related performance fatigability in clinical practice: a systematic review. Eur J Phys Rehabil Med

10. Severijns DZI, Dalgas U, Lamers I, Lismont C, Feys P (2017) The assessment of motor fatigability in persons with multiple sclerosis: a systematic review. Neurorehabil Neural Repair 31(5):19

11. Schwid SR, Thornton CA, Pandya S, Manzur KL, Sanjak M, Petrie MD, McDermott MP, Goodman AD (1999) Quantitative assessment of motor fatigue and strength in MS. Neurology. 53(4):743750

12. Brauers L, Geijen MM, Speth LA, Rameckers EA (2017) Does intensive upper limb treatment modality hybrid constrained induced movement therapy (H-CIMT) improve grip and pinch strength or fatigability of the affected hand? J Pediatr Rehabil Med 10(1):1117

13. Brauers L, Rameckers E, Severijns D, Feys P, Smeets R, Klingels K (2019) Measuring motor fatigability in the upper limbs in individuals with neurologic disorders: a systematic review. Arch Phys Med Rehabil

14. Doix AC, Gulliksen A, Brændvik SM, Roeleveld K (2013) Fatigue and muscle activation during submaximal elbow flexion in children with cerebral palsy. J Electromyogr Kinesiol 23(3):721-726 
15. Fong DT, Yam KY, Chu VW, Cheung RT, Chan KM (2012) Upper limb muscle fatigue during prolonged Boccia games with underarm throwing technique. Sports Biomechanics 11(4):441-451

16. Hong T, Zhang X, Ma HJ, Chen Y, Chen X (2016) Fatiguing effects on the multi-scale entropy of surface electromyography in children with cerebral palsy. Entropy 18(5)

17. Xu KS, Mai JN, He L, Yan XH, Chen Y (2015) Surface electromyography of wrist flexors and extensors in children with hemiplegic cerebral palsy. Pm\&R. 7(3):270-275

18. Surakka J, Romberg A, Ruutiainen J, Virtanen A, Aunola S, Maentaka K (2004) Assessment of muscle strength and motor fatigue with a knee dynamometer in subjects with multiple sclerosis: a new fatigue index. Clin Rehabil 18(6):652-659

19. Severijns D, Lemmens M, Thoelen R, Feys P (2016) Motor fatigability after low-intensity hand grip exercises in persons with multiple sclerosis. Mult Scler Relat Disord 10:7-13

20. Severijns D, Octavia JR, Kerkhofs L, Coninx K, Lamers I, Feys P (2015) Investigation of fatigability during repetitive robot-mediated arm training in people with multiple sclerosis. PLoS One 10(7): e0133729

21. De Vet HCT, C; Mokkink, L; Knol, D. Measurement in medicine. Cambridge; 2011

22. Brehm MA, Knol DL, Harlaar J (2008) Methodological considerations for improving the reproducibility of walking efficiency outcomes in clinical gait studies. Gait Posture 27(2):196-201

23. Brehm MA, Scholtes VA, Dallmeijer AJ, Twisk JW, Harlaar J (2012) The importance of addressing heteroscedasticity in the reliability analysis of ratio-scaled variables: an example based on walking energy-cost measurements. Dev Med Child Neurol 54(3):267-273

24. Bland JM, Altman DG (1986) Statistical methods for assessing agreement between two methods of clinical measurement. Lancet (London, England) 1(8476):307-310

25. Molenaar HM, Selles RW, Schreuders TA, Hovius SE, Stam HJ (2008) Reliability of hand strength measurements using the Rotterdam intrinsic hand Myometer in children. J Hand Surg 33(10):1796-1801

26. Molenaar HM, Zuidam JM, Selles RW, Stam HJ, Hovius SE (2008) Age-specific reliability of two grip-strength dynamometers when used by children. J Bone Joint Surg Am 90(5):1053-1059

27. de Vet HC, Terwee CB, Knol DL, Bouter LM (2006) When to use agreement versus reliability measures. J Clin Epidemiol 59(10): 1033-1039

28. Simon-Martinez C, Mailleux L, Ortibus E, Fehrenbach A, Sgandurra G, Cioni G, Desloovere K, Wenderoth N, Demaerel P, Sunaert S, Molenaers G, Feys H, Klingels K (2018) Combining constraint-induced movement therapy and action-observation training in children with unilateral cerebral palsy: a randomized controlled trial. BMC Pediatr 18(1):250

29. Eken MM, Dallmeijer AJ, Houdijk H, Doorenbosch CA (2013) Muscle fatigue during repetitive voluntary contractions: a comparison between children with cerebral palsy, typically developing children and young healthy adults. Gait Posture 38(4):962-967

Publisher's note Springer Nature remains neutral with regard to jurisdictional claims in published maps and institutional affiliations. 